\title{
Recent advances in first principles computations in materials research
}

\author{
R. Ramprasad $\cdot$ V. Kumar $\cdot$ L. R. C. Fonseca $\cdot$ \\ B. R. Tuttle
}

Received: 11 July 2012/ Accepted: 11 July 2012/Published online: 24 July 2012

(C) Springer Science+Business Media, LLC 2012

The growing need to understand factors that govern the properties of materials, rapid strides in theory, and the wide availability of scientific software and computing resources has lead to an explosion of theoretical and computational studies of materials. While several classes of materials modeling methods have reached high levels of maturity, first principles computational methods (the focus of this special issue) occupy a special place in today's portfolio of materials computations. In its most general form, this brand of computations-also referred to as $\mathrm{ab}$ initio computations-involves solution of the quantum

\section{R. Ramprasad ( $\square)$}

Materials Science and Engineering Program, Department of Chemical, Materials, and Biomolecular Engineering, University of Connecticut, 97 N. Eagleville Rd., U-3136, Storrs, CT 06269-3136, USA

e-mail: rampi@uconn.edu

\section{Kumar}

Dr. Vijay Kumar Foundation, 1969 Sector 4,

Gurgaon 122001, Haryana, India

e-mail: kumar@vkf.in

\section{R. C. Fonseca}

Center for Semiconductor Devices, State University

of Campinas, Campinas, SP, Brazil

e-mail: fonsecalrc@yahoo.com

\section{R. C. Fonseca}

Rua Prof. Otto Cirne 81/504, Belo Horizonte,

MG 30380-610, Brazil

B. R. Tuttle

Physics Department, Pennsylvania State University, Erie, PA, USA

e-mail: brt10@psu.edu

B. R. Tuttle

6141 Bridlewood Dr, Fairview, PA 16415, USA mechanical equations for complex many-nuclei, manyelectron materials systems. The fundamental nature of this essentially parameter-free paradigm makes this approach extremely versatile, allowing for a high-fidelity treatment of a very wide class of materials at any level of dimensionality.

Approximations perforce need to be made to render these computations tractable. One class of approximations relates to mapping the true (formidable) interacting manyelectron system (moving in the field of the nuclei and other external potentials) to an equivalent (manageable) noninteracting many-electron system (moving in the field of the nuclei, other external potentials, and a potential that represents the electron-electron interactions). This transformation of the interacting system to a non-interacting one, and the possibility of expressing the quantum mechanical part of the electron-electron interaction (as well as all other properties) as a functional of the electron density, forms the core of the density functional theory (DFT) - the most popular flavor of first principles computations. Although the exact functional form of the quantum mechanical part of the electron-electron interaction (also referred to as the exchange-correlation interaction) is not known, our ability to derive reasonable approximations to this functional has made DFT an enormously practical tool within present day materials research. The practicality of modern DFT computations has also been steadily improving with time. As a reference point, we note that a $\$ 10,000$ computer system in the 1980 s, 1990 s, and 2000 s could handle with ease systems containing (e.g., within a repeating unit cell) a few atoms, a few tens of atoms, and a few hundred atoms, respectively. The popularity of modern DFT is largely because this method offers the best tradeoff between accuracy and computational cost at the present time. 
This special issue is intended to collect the most recent developments in this rapidly evolving area of first principles computations, and begins with ten full-length review articles followed by topical articles in the areas of "insulating functional materials," "metals, alloys and disordered phases," "magnetism," and culminates with articles that discuss higher spatiotemporal-scale methods that use "DFT-derived approaches."

The first review paper by Hautier et al. [1] provides a remarkable perspective on many success stories (in the form of case studies) of materials discovery and design made possible entirely or largely via first principles computations. Examples include design of materials for highperformance batteries, hydrogen storage, thermoelectrics, multiferroics, and catalysis. This general review is then followed by a survey by Shi et al. [2] of the adsorption of the buckminsterfullerene $\mathrm{C}_{60}$ on close-packed metal surfaces - a classic example of a situation where DFT computations of adsorption, electronic structure, and surface reconstruction have been directly compared with experimental data to arrive at a fundamental understanding concerning adsorption and interface chemistry. In the next article, Singh and Yakobson [3] review hydrogen storage in carbon-based sorption materials such as carbon nanotubes and graphene. The chemistry of Kubas bonding, chemisorption, role of catalysts, and kinetic factors are addressed. The next two articles focus on nuclear materials, which are expected to be an important materials research area in the coming decades. Liu et al. [4] review the current stateof-the-art pertaining to DFT studies of nuclear fuel materials, including uranium oxides, carbides, and nitrides, and defect chemistries and atomic transport in these materials. Nguyen-Manh et al. [5] then provide an overview of methods to understand the phase stability and radiation effects in structural materials for future fusion power-plant applications. The review series moves next to two articles pertaining to oxide thin films and interfaces involving oxides. The first of these by Zhu et al. [6] deals with $\mathrm{HfO}_{2}$, a material expected to gradually replace $\mathrm{SiO}_{2}$, the main dielectric material used in semiconductor transistor technology for the last four decades. The second article by Garrity et al. [7] surveys the current state of understanding pertaining to interfaces (with $\mathrm{Si}$ ) involving complex oxides such as $\mathrm{SrTiO}_{3}, \mathrm{LaAlO}_{3}$, etc., all of which are potential contenders for next-generation high-performance electronic devices. The review article by Scheicher et al. [8] documents the contributions made by first principles methods in molecular genetics, e.g., DNA sequencing, an area in which such computational methods are only now beginning to emerge, but are believed to become increasingly prominent. The set of review articles culminates with two important "prospective" contributions dealing with beyond-DFT methodologies: Ren et al. [9] provide a comprehensive historical and scientific sketch of the random phase approximation and its role in the proper treatment of the electron correlation interaction, and Faber et al. [10] document the promise that many-body perturbation theory methods hold for current and future electronic structure calculations by taking electron-phonon interactions and charge-transfer excitations in organic systems as examples.

Next, the special issue takes the reader to specific topical contributions. The first area, one in which modern first principles methods have made enormous headway, is the field of insulating functional materials. The first article by Mishra et al. [11] addresses the properties of native point defects in indium phosphide, a semiconductor of interest to the optoelectronics industry. Specifically, the authors report calculations identifying the most common native defects along with their respective defect levels. The next three articles address the various properties of transition metal oxides with insights for functional memory devices, photo-catalysts, and optoelectronics, respectively. MagyariKope et al. [12] report calculations of oxygen vacancy ordering effects in candidate oxides for variable resistance and memory devices. Prasai et al. [13] compare and contrast the electronic properties of amorphous and crystalline forms of $\mathrm{TiO}_{2}$ providing guidance for photocatalytical applications. Kamisaka et al. [14] investigate electron trapping in $\mathrm{Nb}$-doped $\mathrm{TiO}_{2}$ crystals and are able to correlate the electronic structure of these materials with their physical structure. The next two articles address properties of $\mathrm{CeO}_{2}$, a promising material for solid oxide fuel cells. Dholabhai and Adams [15] determine the oxygen ion conductivity as a function of temperature and doping concentration. The article by Peles [16] applies DFT augmented with Hubbard parameters to gain insight into the reduction-oxidation reactions in various $\mathrm{CeO}_{2}$-based oxides. The contribution by Joshi et al. [17] explores the role of pressure on the electronic properties of $\mathrm{MgO}$ polymorphs. The next two papers address materials employed in lithium ion battery applications. The first paper by Kim [18] reports the properties of doped $\mathrm{LiNiO}_{2}$, a promising cathode material. The second paper by Radin et al. [19] finds that the surface properties of $\mathrm{Li}_{2} \mathrm{O}_{2}$ are important for understanding the capabilities of $\mathrm{Li}$ oxygen batteries. Tsetseris and Pantelides [20] illustrate the importance of defects for the uptake of hydrogen on graphene to form the insulating material, graphane. Continuing the foray into nanostructured materials, the paper by Pilania and Ramprasad [21] reports the dielectric permittivity of $\mathrm{PbTiO}_{3}$ nanowires. The last paper of this section by Dong and Alpay [22] presents an exhaustive study of the piezoelectric and elastic properties of $\operatorname{In}_{\mathrm{x}} \mathrm{Ga}_{1-\mathrm{x}} \mathrm{N}$ solids.

The next group of articles falls under the general rubric of metals, alloys, and disordered phases. Leitao et al. 
[23] provide a critical survey of studies involving the (100) and (111) surfaces of $\mathrm{Cu}_{3} \mathrm{Al}$ with special focus on segregation of atomic species to the surface under various chemical conditions. Methods to compute solubility products and precipitation (for e.g., $\mathrm{NbC}$ ) in alloyed steels are then presented in the article by Klymko and Sluiter [24]. The importance of electron correlation and relativistic effects in systems such as $\mathrm{Cr}_{2} \mathrm{AlC}$ and $\mathrm{Ta}_{2} \mathrm{AlC}$ (also referred to as MAX phases) is then evaluated by Sun et al. [25]. Wang et al. [26] then discuss factors that control the stability of $\mathrm{Ti}_{1-x} \mathrm{Al}_{x} \mathrm{~N}, \mathrm{Ti}_{1-x} \mathrm{Zr}_{x} \mathrm{~N}$, and $\mathrm{Zr}_{1-x} \mathrm{Al}_{x} \mathrm{~N}$ alloys, which are popular coating materials. This topic is concluded with two articles on amorphous (or disordered) systems. Tian et al. [27] present a first principles molecular dynamics study of $\mathrm{Ni}_{62.5} \mathrm{Nb}_{37.5}$ bulk metallic glasses, and Sun [28] presents the structure of amorphous $\mathrm{Ge}_{2} \mathrm{Sb}_{2} \mathrm{Te}_{5}$, an important material for phase change memory applications.

We then move on to the general topic of magnetism. The first of the articles in this category by Shinde et al. [29] deals with the impact of Gd-doping on the atomic and electronic structure and the magnetic properties of Gd-doped gold clusters. Mao et al. [30] explore the role of ferromagnetism (and temperature) on the $\mathrm{Ni} / \mathrm{Ni}_{3} \mathrm{Al}$ interfacial free energy. The next two studies involve site substitution in materials with interesting magnetic applications. Mukherjee et al. [31] investigate the effect of $\mathrm{B}$ site substitution in $\mathrm{CaCu}_{3} \mathrm{~B}_{4} \mathrm{O}_{12}$, perovskite oxides with potential for spintronics applications. Saini [32] uses an all-electron method to study the role of $\mathrm{f}$-states in rare earth tri-florides, materials with applications to magneto-optical recording devices. The next contribution by Palandage et al. [33] involves a first principles study of electron pairing and magnetism in FeTe-based systems. The final article in this category by Galanakis and Sasioglu [34] evaluates exchange interactions, spin waves, and the Curie temperature in $\mathrm{L}_{1}{ }^{-}$and $\mathrm{L1}_{2}$-type systems such as Heusler alloys and systems containing Pt and Co.

Finally, we note that although first principles methods are versatile, accurate, and practical for investigating many materials and phenomena, several important phenomena involve large length-scales (e.g., involving hundreds of thousands of atoms) and time-scales (e.g., nanoseconds or higher). Such situations are, at the present time, beyond the reaches of first principles computations and are best treated using model Hamiltonians, force fields, or inter-atomic potentials parameterized by means of first principles data. Furthermore, many situations require the organized extraction of knowledge, insights, and trends from first principles, or first principles-derived data. The last three articles provide examples of such strategies. Banerjee et al. [35] present a hybrid quantum mechanics/molecular mechanics approach aimed at a study of gold clusters protected by surfactants, Chernatynskiy et al. [36] provide a critical assessment of various flavors of inter-atomic potentials used in studies of $\mathrm{UO}_{2}$ fuels aimed at thermal conductivity calculations, and Sukumar et al. [37] provide insights into how cheminformatics strategies such as quantitative structure property relationships may be used to extract knowledge from first principles (or other) data. These methods are synergistic to first principles methods and are seen to be essential to bridge the (inevitable) gaps between situations realistically accessible by means of purely first principles methods and complex real-life situations.

The guest editors are delighted to have had this opportunity to coordinate the creation of this special issue. They would like to thank the Deputy Editor-in-Chief Professor M. Aindow and the Editor-in-Chief Professor C. B. Carter for their support and guidance on critical matters during the entire course of this project. Special thanks must also go to the Journal of Materials Science staff, especially Mrs. Saraswathi Sabapathy, who diligently handled the communications and copy-editing of the manuscripts.

\section{References}

1. Hautier G et al (2012) J Mater Sci. doi:10.1007/s10853-0126424-0

2. Shi X et al (2012) J Mater Sci. doi:10.1007/s10853-012-6361-y

3. Singh A, Yakobson B (2012) J Mater Sci. doi:10.1007/ s10853-012-6551-7

4. Liu X -Y et al (2012) J Mater Sci. doi:10.1007/s10853-0126471-6

5. Nguyen-Manh D et al (2012) J Mater Sci. doi:10.1007/s10853012-6657-y

6. Zhu H et al (2012) J Mater Sci. doi:10.1007/s10853-012-6568-y

7. Garrity K et al (2012) J Mater Sci. doi:10.1007/s10853-0126425-Z

8. Scheicher R et al (2012) J Mater Sci. doi:10.1007/s10853-0126671-0

9. Ren X et al (2012) J Mater Sci. doi:10.1007/s10853-012-6570-4

10. Faber $\mathrm{C}$ et al (2012) J Mater Sci. doi:10.1007/s10853-012-6401-7

11. Mishra R et al (2012) J Mater Sci. doi:10.1007/s10853-0126595-8

12. Magyari-Kope B et al (2012) J Mater Sci. doi:10.1007/s10853012-6638-1

13. Prasai B et al (2012) J Mater Sci. doi:10.1007/s10853-0126439-6

14. Kamisaka H et al (2012) J Mater Sci. doi:10.1007/s10853-0126491-2

15. Dholabai P, Adams J (2012) J Mater Sci. doi:10.1007/s10853012-6398-y

16. Peles A (2012) J Mater Sci. doi:10.1007/s10853-012-6423-1

17. Joshi K et al (2012) J Mater Sci. doi:10.1007/s10853-012-6521-0

18. Kim Y (2012) J Mater Sci. doi:10.1007/s10853-012-6299-0

19. Radin $M$ et al (2012) J Mater Sci. doi:10.1007/s10853-0126552-6

20. Tsetseris L, Pantelides S (2012) J Mater Sci. doi:10.1007/s10853012-6447-6

21. Pilania G, Ramprasad R (2012) J Mater Sci. doi:10.1007/s10853012-6411-5

22. Dong L, Alpay S P (2012) J Mater Sci. doi:10.1007/s10853-012-6351-0

23. Leitao A et al (2012) J Mater Sci. doi:10.1007/s10853-0126468-1 
24. Klymko T, Sluiter M (2012) J Mater Sci. doi:10.1007/s10853012-6590-0

25. Sun W et al (2012) J Mater Sci. doi:10.1007/s10853-012-6609-6

26. Wang A et al (2012) J Mater Sci. doi:10.1007/s10853-011-6223-Z

27. Tian H et al (2012) J Mater Sci. doi:10.1007/s10853-012-6306-5

28. Sun Z (2012) J Mater Sci. doi:10.1007/s10853-012-6607-8

29. Shinde $P$ et al (2012) J Mater Sci. doi:10.1007/s10853-0126632-7

30. Mao Z et al (2012) J Mater Sci. doi:10.1007/s10853-012-6399-x

31. Mukherjee S et al (2012) J Mater Sci. doi:10.1007/s10853-012$6530-\mathrm{z}$
32. Saini S (2012) J Mater Sci. doi:10.1007/s10853-012-6459-2

33. Palandage K et al (2012) J Mater Sci. doi:10.1007/s10853-0126608-7

34. Galanakis I, Şaşığlu E (2012) J Mater Sci. doi:10.1007/s10853012-6285-6

35. Banerjee S et al (2012) J Mater Sci. doi:10.1007/s10853-0126356-8

36. Chernatynskiy A et al (2012) J Mater Sci. doi:10.1007/s10853011-6230-0

37. Sukumar N et al (2012) J Mater Sci. doi:10.1007/s10853-0126639-0 